\title{
Database-Assisted Distributed and Cloud-Based Access Methods for Unlicensed and Radar Bands
}

\author{
Zaheer Khan, Janne J. Lehtomäki, Rafael Aguilar, Risto Vuohtoniemi, Ekram Hossain, Luiz A. DaSilva, and Alan \\ Marshall
}

\begin{abstract}
This paper focuses on the design of methods which enable unlicensed devices to use the frequency spectrum already allocated to rotating radar systems without causing interference to those radars. We first present a database-assisted spectrum sharing/co-existence mechanism for radar spectrum bands. This mechanism takes into account the real spectrum usage behavior of different radars, and is also implemented by prototyping a real-time rotating radar beam emulator, a radar spectrum database using MySQL software, and spectrum access algorithm on Wireless Open Access Research Platform. Then we propose a cloud-based unified channel access (UCA) method and a distributed UCA method for co-existence of multiple competing users in unlicensed and rotating radar spectrum bands. We model the UCA problem as a game and propose iterative methods (i.e., algorithms) to obtain the solution of the game. We study the stability of the proposed methods under several different scenarios. We show that the proposed UCA game is a potential game, and also show that the proposed methods guarantee convergence to a Nash Equilibrium. Our results show that the cloud-based method allows fast convergence and can achieve performance that is close to optimal solution.
\end{abstract}

Index Terms-Spectrum sharing, unlicensed channels, radar channels, game theory, potential games, cloud-assisted, distributed spectrum access, MySQL database, prototype.

\section{INTRODUCTION}

\section{A. Background and Contributions}

In response to the spectrum deficit, the wireless industry has set in motion several initiatives that aim at: 1) exploring new models for spectrum sharing [1]; and 2) exploring new infrastructure models that use cloud-assisted solutions to provide flexible spectrum management and advanced network coordination [2], [3]. In terms of spectrum sharing, design of new models of shared access (SA) between rotating radars and wireless communications has generated particular interest. This is due to the reason that radar systems consume large amount of highly desirable spectrum below $6 \mathrm{GHz}$ [4], [5]. For example, potential candidate radar channels for sharing are between $960-1400 \mathrm{MHz}, 2700-3650 \mathrm{MHz}$, and 5000$5850 \mathrm{MHz}$, since different wireless technologies such as LTE, WiMAX, and WLAN can support operation in one of these bands.

Dynamic frequency selection (DFS) enabled unlicensed devices currently share spectrum with radars in the $5 \mathrm{GHz}$ band. However, in this method, it is challenging to detect with close to $100 \%$ probability in a way that also minimizes

Z. Khan is with the University of Liverpool, UK, and part of this work was done while he was with the University of Oulu, Finland, J. J. Lehtomäki, and R. Vuohtoniemi are with the University of Oulu, Finland. E. Hossain is with the University of Manitoba, Canada. L. A. DaSilva is with the Trinity College Dublin, Ireland. A. Marshall is with the University of Liverpool, UK. the DFS false alarm rate [5]. DFS is also not an efficient mechanism in the search for spectrum opportunities, as it requires long channel availability check time periods, and long non-occupancy periods. To address the challenge of spectrum sharing in radar spectrum, regulatory bodies have generated intense interest in the design of database technology that can make spectrum sharing feasible not only in TV White space (TVWS) bands but also in new bands, such as radar bands [4], [6], [7]. Although the basic requirements for database services have been established in different works [8], [9], many of the details of spectrum databases and their use for spectrum sharing need to be worked out.

Spectrum measurement campaigns that obtain reliable spectrum occupancy results for radar systems are crucial for the design of database-assisted spectrum sharing models. To take this into account we ran a measurement campaign to assess the spectrum usage behavior of different rotating radar systems in Finland. Our recent works in [10], [11] describe the results of our measurement campaign. Using the data we collected, in this paper, we first present a database-assisted shared spectrum access mechanism between the rotating radars and a secondary network that poses low overhead and requires minimum interaction between the two types of user. In order to realize a complete real-time SA system, the proposed method is also implemented by prototyping a real-time rotating radar beam emulator, a radar spectrum database using MySQL server software, and spectrum access algorithm on Wireless Open Access Research Platform.

In practice, AP deployments in unlicensed and SA channels can be distributed, as a user can individually choose to deploy an AP at his/her home or at some other place, or coordinated, as hospitals, universities, or businesses choose to deploy a network of hundreds of APs within their premises. Our work proposes solutions for both scenarios and designs distributed and cloud-assisted unified channel access (UCA) techniques. In particular, we focus on the design of UCA techniques for multiple competing users that seek to optimize their spectrum usage and operate in frequency channels where some channels are for unlicensed access and other channels are for sharing with the rotating radars. Game theory provides a natural framework for modeling such competing interests of wireless users. We model the network of multiple users as a UCA strategic game in which the users attempt to optimize their utility. We consider several different network scenarios and show that the UCA game is a generalized ordinal potential game under certain scenarios, and an exact potential game in other scenarios. Such games guarantee the existence of at least one pure Nash Equilibrium (NE) (i.e., local maximum). 
Recent advances in cloud-based technologies have given a platform for the design of systems that can handle and efficiently process computationally heavy tasks in different application areas, such as cloud-based management of multiple wireless systems [3], [12], autonomous cloud-based network for driving [13], and cloud-based decision making for selection of servers [14], [15]. We present a cloud-based system for UCA in which the cloud has access to a spectrum database as well as local spectrum usage reports from users. The cloudbased system is a software-based entity and is illustrated in Fig. 1. The three main characteristics of the proposed cloud-assisted wireless system are: i) centralized computing of a stable and efficient UCA solution for multiple wireless users, ii) reconfigurability of spectrum resources, iii) and collaborative communications.

In our model, the cloud has no power to enforce the solution, and the users would agree to such a proposal only if it were an NE. By adding a low-overhead cloud-based information exchange system to the UCA game, we ensure that the users always reach an NE in a few rounds. The proposed cloudbased UCA method requires no more than a total of $2 N$ best response updates to reach an $\mathrm{NE}$, where $N$ is the number of players. In game theory, the best response is the action which produces the most favorable outcome for a player, taking other players' actions as given.

Different from the cloud-based method, in the proposed distributed method each AP takes autonomous channel selection decisions, i.e., it does not take assistance from the cloud in terms of channel selections. We compare the performance of the cloud-assisted method with the proposed distributed method. We show that, while the performance of proposed cloud-assisted method is not strongly affected by an increase in the number of competing wireless users, the distributed UCA method does show degradation when the number of users is increased beyond the total capacity of all the available channels. We also find that under the distributed UCA method, when an AP can measure one extra channel apart from the channel it has selected to access, the performance of the proposed UCA method improves significantly. Surprisingly, we also find that when the number of channels that a user can measure is increased beyond a few channels, then the performance of the distributed UCA method starts deteriorating, and results in the worst performance when the user can measure all the channels. This counterintuitive result stems from the increased likelihood that two or more users select the same channel, as will be further explained later in Section VI.

\section{B. Related Literature}

Most existing spectrum sharing studies have focused on the design of channel selection techniques in either unlicensed [2], [16] or in SA bands [17], [18]. Such methods may be inefficient for network operators planning $5 \mathrm{G}$ networks, as the next generation of infrastructures and devices will need to be more flexible to be able to operate in different spectrum bands [19], and exploit multiple radio access schemes [20]. In this context, the design of unified spectrum sharing techniques using which multiple users can flexibly select channels from both unlicensed and SA channels is important. Different from previous works, we consider the problem of UCA in both unlicensed and SA radar bands. In particular our proposed SA method takes into account real spectrum usage of rotating radar systems.

The problem of multi-user resource allocation process for a single unlicensed channel where a centralized mediating authority receives local interference reports from the links and instructs them on spectrum usage has been investigated in different works (see [21], and the references therein). Dynamic spectrum usage in a single radar channel is the topic of [17] and [18]. These works focus on the use of temporal sharing in a radar channel which exploits spectrum opportunities both in the spatial and temporal domains.

Different from [21], [17] and [18], our work focuses on the design of UCA techniques for scenarios where multiple frequency channels are available and some of these channels are for unlicensed access use, and other channels are for SA with rotating radar systems. The works in [22], [23] investigate the problem of database-assisted SA in TV White Space. A social group utility maximization for database assisted SA in TV White Space is the topic of [24]. The proposed techniques in [22]-[24] are designed for co-existence of secondary users under primary user constraints in TV White Space. These techniques cannot be applied/compared to the problem studied in this work as they do not take into account the spectrum usage behavior of rotating radars. Moreover, the techniques proposed in [22]-[24] are different from our work as we consider channel selection under both unlicensed access and SA scenarios.

In [25], an online algorithm for distributed channel and bandwidth selection in unlicensed channels is presented. Although, the algorithm relies on airtime utilization measurements in channel/bandwidth selection, however, the work mainly focuses on finding efficient variable-width spectrum configurations (between $5 \mathrm{MHz}$ to $40 \mathrm{MHz}$ ) for WLANs in unlicensed channels. Our proposed methods do not consider that the users are capable of dynamic bandwidth selection. To analyze the conflict among multiple self-motivated users, many research papers have been published that have utilized the framework of potential games in the context of channel selection (see [26], and references therein). However, to our knowledge, no existing research work exists using potential games to analyze equilibrium properties of a wireless network in which competing users can select a channel from both unlicensed and SA radar channels. Moreover, our work is also different from the previous works on potential games because our proposed utility function takes into account airtime utilization in channel selection. This is motivated by the fact that many recent works [27]-[30] have shown that airtime utilization in wireless local area networks (WLANs) determines the resulting throughput performance and access latency of the WLANs. 


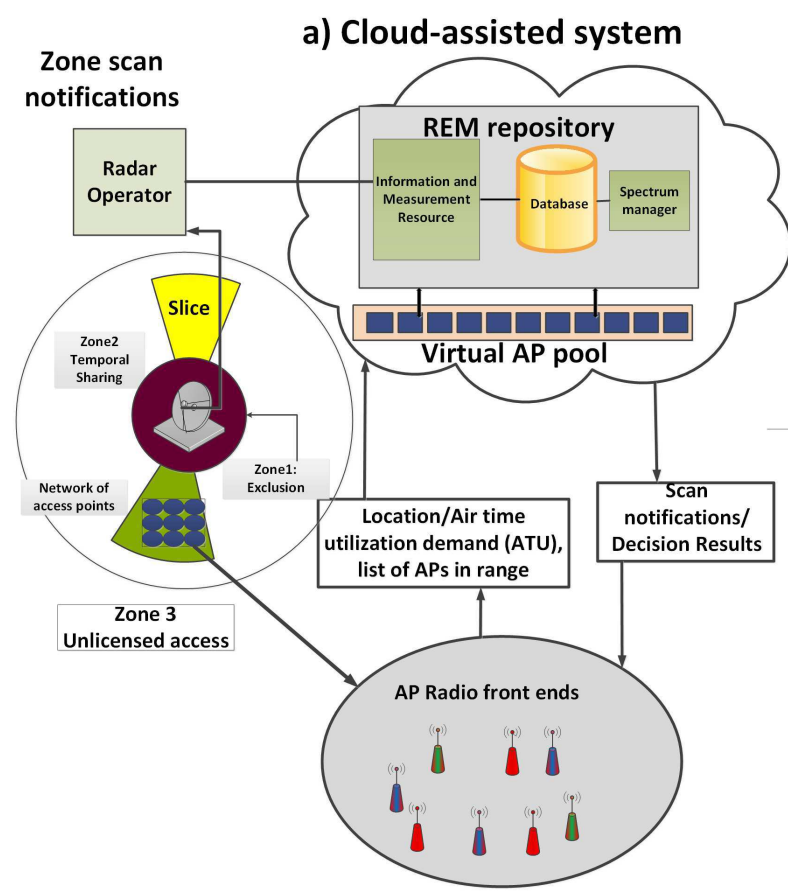

\section{b) Distributed system}

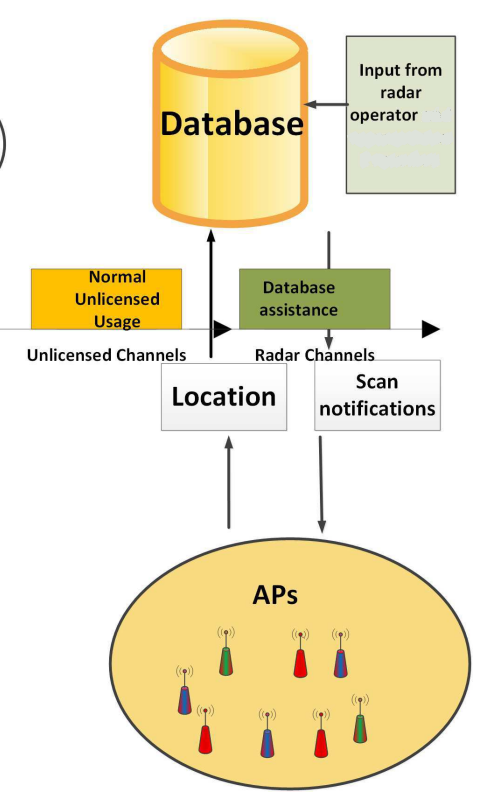

Fig. 1. A simple systems design illustrating different geographic zones, the cloud-based and distributed framework and how each interact with the access points.

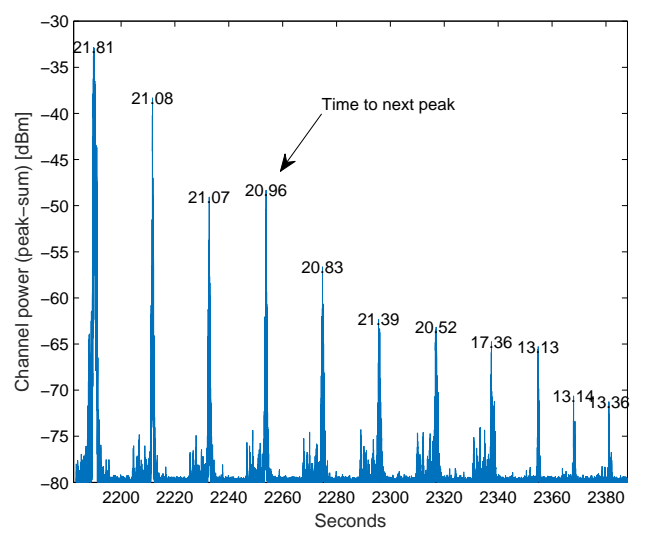

Fig. 2. Example results of our measurements of a weather radar spectrum usage.

\section{Database-Assisted Spectrum Sharing With ROTATING RADARS}

\section{A. Rotating Radars and Their Spectrum Usage}

The rotating radars that operate in different bands have highly directional rotating antennas and provide coverage over a large area (e.g., they can have a range of up to 50$200 \mathrm{~km}$ ). Examples of rotating radars include weather radar systems in the $5 \mathrm{GHz}$ band, air surveillance radar systems, and ground surveillance radar systems. These radars transmit a narrow beam and they perform more listening than talking. For example, a weather radar may emit a pulse for $2 \mu$ s then listen for approximately $2 \mathrm{~ms}$. They rotate to scan horizontally 360 degrees, and some of them also tilt vertically.
To better understand the operating principles of various radar systems, and to determine their spectrum usage patterns, we measured spectrum usage behavior of three different ground-based fixed rotating radar systems at different locations near Oulu, Finland. Detailed results of our measurements campaign, their explanations, and a discussion of challenges for existing sharing techniques in the radar bands can be found in [10], [11]. In Fig. 2, from our measurements campaign, we present an example result of spectrum usage by a weather radar system that is located near the city of Oulu, Finland. It can be seen from Fig. 2 that at a given location there are pauses between the radar scan pulses that vary from 13.1 seconds to 21.1 seconds. This offers the potential of temporally sharing the spectrum with the radar system. However, the fact that the scan pulse interval varies from 13.1 seconds to 21.8 seconds shows that the weather radar's scan patterns are quasi-periodic. This poses a challenge for existing theoretical sharing models which are designed based on the assumption that the scan patterns are periodic [18]. Moreover, it can be also seen in Fig. 2 that the received peak power is not constant. The reason for this power variation is that the radar scans horizontally 360 degrees at different vertical angles. The highest peaks in the figure are obtained when the radar directs its beam downward to the measurement location. This significant variation in received signal of the radar poses a challenge for sensingbased spectrum sharing techniques.

\section{B. Motivation and Explanation for the Proposed Spectrum Access Framework}

In our proposed database-assisted framework, the radar's surrounding area is divided into three geographic zones and several slices (see Fig. 1 for an illustrative example). Each 


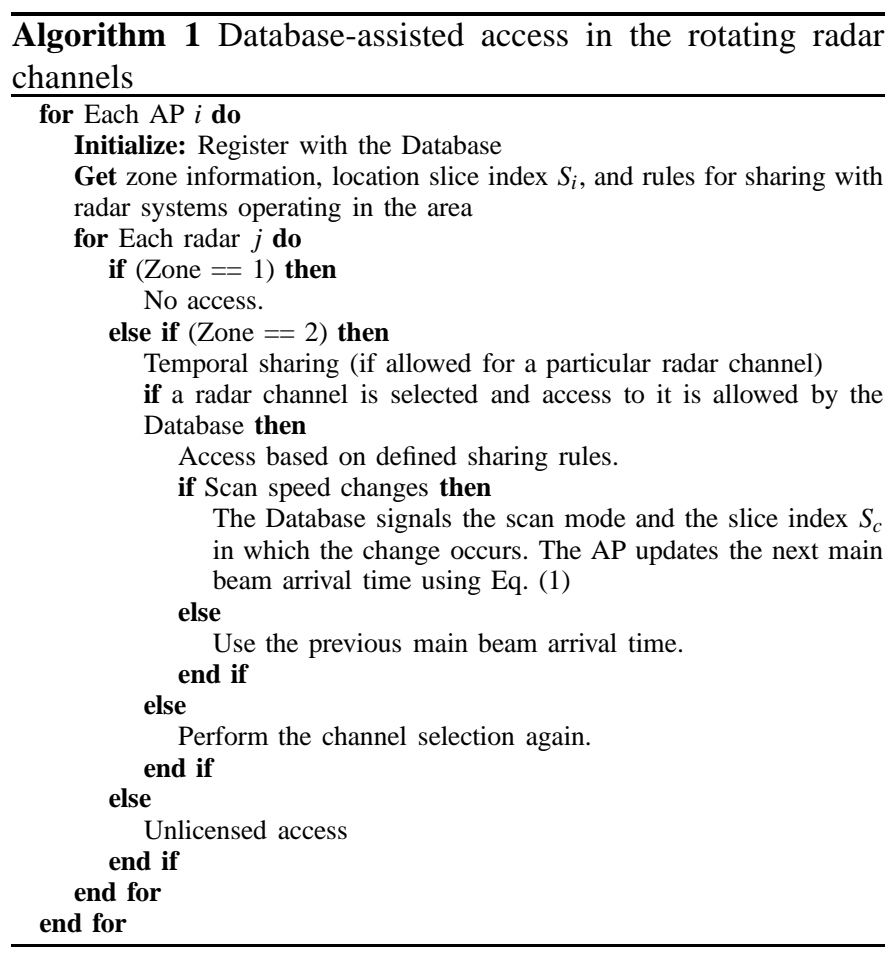

zone defines a different operating mode for an AP. Each slice $S$ is defined by its angular width $\theta_{S}$, which in turn has the same value as the radar beamwidth. Let $N_{S}$ represent the total number of slices which is given by $\left\lfloor N_{s}=360 / \theta_{S}\right\rfloor$. The time the radar's main beam spends on each slice is $T_{S}=\theta_{S} / R$, where $R$ is the scan speed in degrees/sec. Algorithm 1 describes the main steps followed by a wireless AP to access the rotating radar's spectrum in which shared spectrum access is allowed.

Exclusion zone analyses and methodology for sharing with various radar systems are actively being researched within the regulatory bodies. In [31], and [32], both the Federal Communications Commission (FCC) and the National Telecommunications and Information Administration (NTIA) have laid detailed groundwork relating to the calculation of exclusion zone distances around different rotating radar systems, such as shipborne and ground-based radar systems. The results of the studies in [31], and [32] suggest, a protection zone of at least $3 \mathrm{~km}$ is required around the perimeter boundary of the installations where the ground-based radars are deployed. In our work, we target close to zero probability of interfering with the radar system. This is achieved by a zone-based SA approach. In our proposed Algorithm 1, the different zones around a radar are modeled as follows. In Zone 1 (which is a region covering $3 \mathrm{~km}$ with a radar station at its centre), opportunistic secondary operation is strictly forbidden since it can cause interference on the incumbent radar. In Zone 2, temporal sharing (TS) can take place for radar systems that authorize such sharing mechanism. In TS, the users can transmit every time the radar's main beam is pointing in another direction. The users stay quiet during the $T_{S}$ interval and also during the $T_{g}$ guard intervals, i.e., $\left(T_{g}+T_{s}+T_{g}\right)$ sec. The use of guard intervals before and after the main beam arrival ensures that the user does not interfere with the main beam pulse or with its side lobes. Moreover, to provide further protection, a network of sensor devices called environment sensing capability (ESC) is deployed at the boundary of Zone 1. The detailed advantages of deploying ESC near exclusion zone are shown in our work [33]. The ESC is used to detect aggregate received signal strength. When the received signal strength exceeds a critical threshold value (defined by a regulatory body), some APs in the area are instructed to move to another channel to avoid any possibility of harmful interference. In Zone 3 (which is typically 50 to $200 \mathrm{~km}$ away from a radars' location), the users are free to use the spectrum, as they are outside the interference area of the radar.

Due to the arbitrary change in scan speeds of some radar systems, such as weather radars, there can be some uncertainty in when the radar will direct its main beam to the AP's location. In the proposed framework, this challenge is addressed as follows: When there is a change from fast to slow or slow to fast scan speed, the database signals the scan mode change and the slice in which the change occurred, so that the next pulse arrival time can be calculated by the AP as follows:

$$
T_{A}=\left\{\begin{array}{l}
\left(\left|S_{i}-S_{c}\right| \times T_{B}\right)+\left(\left(N_{s}-\left|S_{i}-S_{c}\right|\right) \times T_{N}\right), \text { when } S_{c} \geq S_{i} \\
\left(\left|S_{i}-S_{c}\right| \times T_{N}\right)+\left(\left(N_{s}-\left|S_{i}-S_{c}\right|\right) \times T_{B}\right), \text { when } S_{c}<S_{i}
\end{array}\right.
$$

where $S_{i}$ is the slice index in which the $i$ th AP is located, $S_{c}$ is the slice index in which the speed change occurred, $T_{B}$ is the previous time the radar's main beam spends on each slice, and $T_{N}$ is the time the radar's main beam now spends on each slice.

It is important to note that our proposed SA method does not require highly-accurate location information of a user as location information is only used to determine in which zone/slice a user is located. In practice, the considered radar systems coverage is around several $\mathrm{km}$ (typically from 50 to $200 \mathrm{~km}$ ), which means a zone around a radar system can span from a few $\mathrm{km}$ to several $\mathrm{km}$. Also, a slice can span from a few to several hundred metres. This in turn means that for a user with slow to medium velocity some delay in location updates can be tolerated.

\section{Information Exchange Overhead}

Using the proposed framework, a secondary network needs to interact with the database only under two scenarios: 1) Before initiating the APs, a spectrum sharing database is queried about the zone information. 2) When the radar scan speed is changed, the database signals the scan mode (fast or slow scan mode), and the slice index $S_{c}$ in which the speed change occurred.

\section{Implementation Using MySQL Software and WARP}

In Fig. 3, we illustrate the block diagram of the software/hardware implementation model of the proposed database-assisted spectrum access method. We have used the measurement-based radar spectrum usage data to implement a real-time rotating radar beam emulator. The radar beam emulator is implemented in Matlab using the Matlab Mapping Toolbox. A screenshot of the emulator is shown in Fig. 3. The radar beam emulator is connected to the spectrum database server which is implemented using the MySQL server software. The database stores user IDs and their 


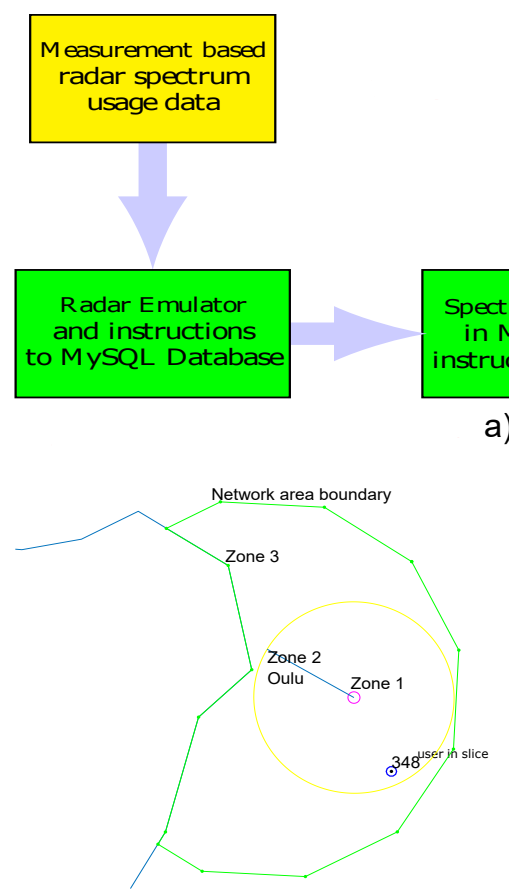

b)

a)
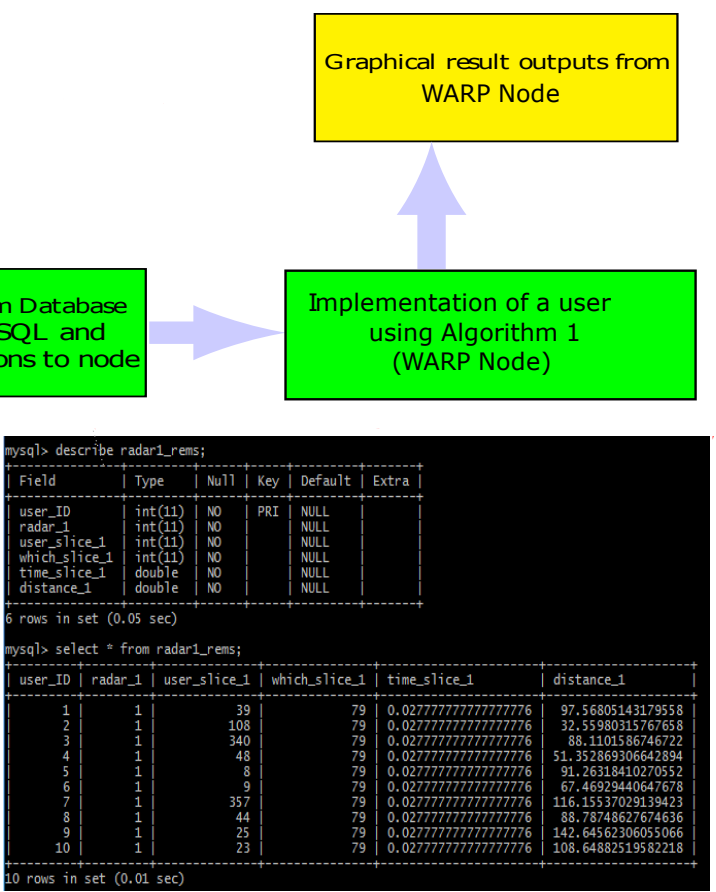

c)

Fig. 3. The block diagram of the software/hardware implementation model of the proposed Algorithm 1, and screenshots of the radar beam emulator and MySQL database.

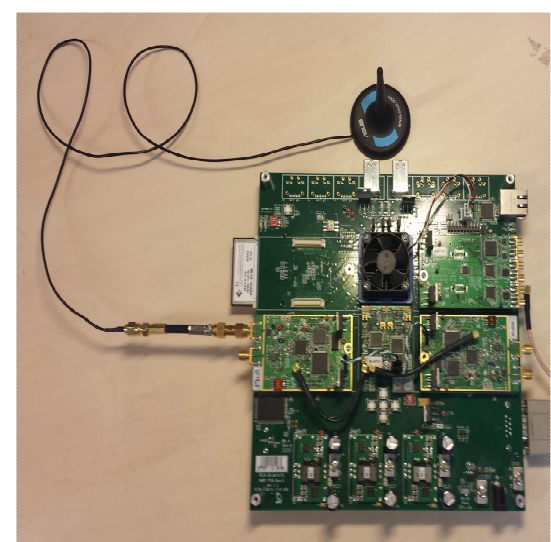

(a)



(b)

Fig. 4. a) A WARP FPGA board used in the implementation. b) Real-time events showing successful implementation of the proposed Algorithm 1.

latitudes/longitudes information. Based on this information, it calculates zone information, location slice index $S_{i}$, and rules for sharing as explained in Algorithm 1. A screenshot of the MySQL database is shown in Fig. 3. The users obtain the rules of spectrum sharing from the database. A user obtaining the rules of information and utilizing them to access the spectrum is implemented using the Wireless Open Access Research Platform (WARP) FPGA board (see Fig. 4a). The availability of open source reference design files related to WARP boards and ease of their modification have motivated us to use WARP boards to prototype our proposed SA method. At the heart of this design is a Xilinx Virtex-family Pro FPGA [34]. This family of FPGAs is very well suited for the real- time DSP-intensive operations required by Algorithm 1. Realtime events showing successful implementation of Algorithm 1 on the WARP node are illustrated in Fig. 4b. The WARP node sends real-time event information to the Matlab via serial communication port which are plotted in Matlab in real time.

In the following sections, we will present the UCA methods for spectrum sharing across unlicensed and radar bands, which use the database-assisted spectrum sharing mechanism presented above. 
III. System AND GAME MOdel For Unified SPectrum ACCESS

\section{A. Network Model}

The system model is illustrated in Fig. 1. We consider a network of $N$ Access Points (APs) deployed independently. The set of channels is $\mathcal{M}=\{1,2, \cdots, M\}$, the set of unlicensed channels is $\mathcal{M}_{u}=\left\{1,2, \cdots, M_{u}\right\}$, and the set of rotating radar channels is $\mathcal{M}_{r}=\left\{M_{u+1}, M_{u+2}, \cdots, M_{u}+M_{r}\right\}$, where $\mathcal{M}_{u} \cup$ $\mathcal{M}_{r}=\mathcal{M}$. An AP can use the unlicensed channels based on typical unlicensed usage rules, and is allowed to make use of the radar channels based on the coexistence mechanism presented in Section II.

It is shown in [27], [28] that the airtime utilization in wireless local area networks (WLANs) determines the resulting throughput performance and access latency of the WLANs. The works in [28]-[30] designed and implemented methods to measure airtime by an AP. The detailed benefits of using airtime as a performance metric in WLANs can be found in [30]. This, along with other recent works, such as the works in [35], motivate us to focus on total airtime utilization of an AP as performance metric.

Let $A^{k}$ represent the total normalized airtime that can be utilized by APs on channel $k$. Note that for an unlicensed channel $A^{k}=1$, and for a radar channel $A^{k}=1$ in Zone 3, $A^{k}<1$ in Zone 2 , and $A^{k}=0$ in Zone $1 . A_{d, i}$ denotes the normalized total airtime demand or total load experienced by an AP $i$ (for its connected devices to achieve their target application throughput levels). The normalized expected airtime obtained by an AP $i$ on a channel when it contends for access in the presence of other neighboring APs which utilize the same channel is given by [36]:

$$
A_{o, i}= \begin{cases}A_{d, i}, & \text { if } \sum_{j \in C^{k}} A_{d, j} \leq A^{k} \\ \min \left\{A_{d, i}, \frac{1}{\left|C^{k}\right|}\right\}, & \text { otherwise }\end{cases}
$$

where $C^{k}$ is the set of all APs that have selected channel $k$. $A_{o, i}$ can be explained as follows. When the sum of total airtime demands is less than the total available airtime in the channel $k$, then the obtained airtime of $i$ is equal to its airtime demand. However, in a channel, when the total airtime utilization demand by multiple APs exceeds $A^{k}$, then the $i$ th can still expect to get its fair share of the airtime which is at most $\frac{1}{\left|C^{k}\right|}$.

\section{B. Game Model}

Let $\mathbb{G}=\left(\mathcal{N},\left(\psi_{i}\right)_{i \in \mathcal{N}},\left(U\left(a_{i}, \mathbf{a}_{-i}\right)\right)_{i \in \mathcal{N}},\left(A_{d, i}\right)_{i \in \mathcal{N}}\right)$ define the unified channel access (UCA) game, where $\mathcal{N}$ is the set of APs (players), $A_{d, i}$ denotes the normalized airtime demand of AP $i$, and $\psi_{i}$ is the strategy set of AP $i$. Each AP can select one of the $M$ channels. In a given round of the game, we also allow APs to opt out of playing by selecting the null strategy, i.e., stay quiet and not select any channel. When an AP selects the null strategy we say it has selected the virtual channel which is denoted by $v=0$. In other words, the set of pure strategies for an AP is $\mathcal{M}_{\mathcal{T}}=\{0\} \cup \mathcal{M}$, and the vector of their action profiles is given by $\mathbf{a}=\left(a_{1}, a_{2}, \cdots, a_{N}\right)$. The utility of AP $i$ when it selects the null strategy is $U\left(a_{i}=0, \mathbf{a}_{-i}\right)=0$. The utility of AP $i$ is given by

$$
U\left(a_{i}, \mathbf{a}_{-i}\right)= \begin{cases}1, & a_{i} \in \mathcal{M}, A_{o, i} \geq A_{d, i} \\ -c, & a_{i} \in \mathcal{M}, A_{o, i}<A_{d, i} \\ 0, & a_{i}=0 .\end{cases}
$$

\section{Algorithm 2 Cloud-based UCA}

\section{2a) Each AP $i$ part}

Initialize: Register with the cloud-based REM.

Get: Rules for sharing with radar systems and channel utilization set using Algorithm 1.

Select: A channel $k \in \mathcal{M}_{a}$ randomly with uniform probability, where $\mathcal{M}_{a}$ is the set of available channels.

Access Channel:

if The selected channel $k$ is a radar channel then

When access is allowed, as rule-based access defined in Algorithm 1. When access is denied stay quiet go to the step Select. else

The selected channel $k$ is an unlicensed channel and access the channel end if

for each round $l$ do

Communicate, Measure: Communicate with users, and Measure $A_{d, i}$, $A_{o, i}$

Report: Report $A_{d, i}$ and $A_{o, i}$ to the REM when $l=1$ and later whenever $A_{d, i}$ and $A_{o, i}$ are changed.

Update Utility:

if $U\left(a_{i}, \mathbf{a}_{-i}\right)<1$ then

Collect best response update $a_{i}$ from the REM and update channel $k$ When $\dot{a}_{i} \neq 0$, go to the step $l=l+1$.

When $a_{i}=0$, i.e., best response channel is the virtual channel), go to the step $l=l+1$ and then to the step Update Utility. else

Utilize channel $k$.

end if

$l=l+1$

end for

Until The Cloud-based REM notifies no channel can be allocated to a particular AP $i$.

\section{2b) Cloud-based REM part}

for each competing AP $i$ and each round $l$ do

Get $A_{d, i}$ and $A_{o, i}$.

Compute the best response channels $B_{i}$ for each $A P i$

Randomly pick a channel $a_{i}$ out of $B_{i}$ channels (when more than one $\left.B_{i}\right)$

Notify the update to AP $i$.

$l=l+1$

end for

Until All APs are allocated or no usable channel can be allocated to remaining APs anymore.

For an AP $i$, the utility of airtime is equal to 1 when the airtime obtained by the AP is greater than or equal to its airtime demand. When its airtime demand cannot be satisfied the utility is $-c$, where $c \ll 1$ is the penalty of being active even though the obtained airtime cannot satisfy the quality of service requirement of the AP's applications, and the utility is zero when the AP stays quiet and does not select any channel.

Remark III.1. In the proposed UCA game, when in a channel the total airtime utilization demand by multiple APs exceeds the channel's total airtime, then an AP $i$ with $A_{d, i} \leq 1 /\left|C^{k}\right|$ can still be satisfied and obtains the utility of one, whereas an AP $j$ with $A_{d, j}>1 /\left|C^{k}\right|$ cannot be satisfied and will obtain a 
utility of $-c$.

\section{Cloud-Based UCA Method}

\section{A. Algorithm}

For the proposed cloud-based UCA method, the APs are connected to a Radio Environment Map (REM) repository entity (illustrated in Fig. 1). The general concept of REM was first introduced in [37]. REM is defined as a collection of network entities which enhances the awareness of access points and/or users by providing them information about their radio environment. Using low-overhead information exchange signals, the entity implements the radar spectrum database decisions and also assists the network of APs to arrive at an equilibrium in few rounds. Algorithm 2 describes the main steps involved in the proposed cloud-based UCA method.

\section{B. Equilibrium and Convergence Properties}

We next show the equilibrium and convergence properties of the proposed cloud-based UCA method under different scenarios. Before presenting the formal proofs we first present some definitions related to the concept of potential games.

Definition 1. Let us consider an action update which only involves one AP $i$ changing its action, while all other APs keep their action choices unchanged. Then we say that an action $a_{i}$ for AP $i$ is a better response update for AP $i$ to the joint action profile $\left(a_{i}, \mathbf{a}_{-i}\right)$ when

$$
U\left(\hat{a}_{i}, \mathbf{a}_{-i}\right)>U\left(a_{i}, \mathbf{a}_{-i}\right) .
$$

Definition 2. An update is a best response update if it improves AP i's payoff to the maximum possible value among all better responses for $A P$ i to the joint action profile $\left(a_{i}, \mathbf{a}_{-i}\right)$.

Definition 3. A pure action profile is a Nash Equilibrium (NE) if no single player can obtain a higher payoff by deviating unilaterally from this profile, i.e.,

$$
U\left(\hat{a}_{i}, \mathbf{a}_{-i}\right) \leq U\left(a_{i}, \mathbf{a}_{-i}\right), \forall \dot{a}_{i} \in \psi_{i}, \forall i \in \mathcal{N} .
$$

Definition 4. A better reply path is a sequence of action profiles in which one AP moves at a time and that AP increases its own utility, whereas a best reply path is a better reply path with the additional requirement that each unilateral deviation is the result of a best response update.

Under the proposed cloud-based method, we consider two different best response update rules for channel selection: (1) utility based best response (UBR) update rule in which a best response update is performed by taking into account only an AP's own utility given in Eq. 3; (2) marginal contribution based best response (MBR) update rule in which a best response update is performed by taking into account an AP's marginal contribution. The marginal contribution $\left(M C_{i}^{k}\right)$ of an AP $i$ with respect to a channel $k$ is defined as follows.

Definition 5. The marginal contribution $\left(M C_{i}^{k}\right)$ of an $A P i$ with respect to a channel $k$ is defined as the total utility with it minus the total utility without it on that channel. Let $\Upsilon\left(C^{k}\right)$ denote the total utility of a set $C^{k}$ of APs which select channel $k$ then $M C_{i}^{k}$ is given by

$$
M C_{i}^{k}=\Upsilon\left(C^{k} \cup i\right)-\Upsilon\left(C^{k} \backslash i\right)
$$

For each best response update rule, we consider two scenarios to initialize the cloud-based channel selections: 1) the scenario in which the cloud respects the initial random channel selection of APs. This scenario is denoted by RIS. The cloud starts with the lowest indexed AP, and it performs best response for APs in increasing order of their indices; and 2) the scenario in which the cloud does not respect the initial random selection. This scenario is denoted by NIS. The cloud initially assigns the null action strategy to each AP. It performs best response for each AP $i$ by sorting the APs in terms of their increasing airtime demands and starts with the lowest airtime demand AP.

The UBR and the MBR rules for an AP lead to the following interesting remark relating to the UCA game.

Remark IV.1. When an AP $i$ updates its channel selection using best response update, and the total airtime utilization in that channel exceeds $A^{k}$ due to the channel selection of AP $i$, then: 1) When the best response update is performed using $M B R$ then the AP i's contribution in that channel is negative; 2) Under the same scenario, when the best response update is performed using UBR then the AP i's utility can be one, when $A_{d, i} \leq 1 /\left|C^{k}\right|$.

Next we show that the proposed cloud-assisted UCA game has at least one NE and the proposed method terminates in an NE. In Fig. 5, we provide illustrations to help understand how the proposed method enables users to converge to an NE for different scenarios.

Theorem IV.1. Under the RIS scenario, the proposed cloudassisted UCA game has at least one pure Nash equilibrium action profile for both $U B R$ and MBR rules.

Proof. We show that the proposed game is a generalized ordinal potential game for both UBR and MBR rules, i.e., the game admits a generalized ordinal potential. A function $\Phi_{g}$ is a generalized ordinal potential for the game $\mathbb{G}$ if AP $i$ obtains a better utility by performing best-response from an action to another one, the potential function increases with this deviation as well, i.e.,

$$
\begin{aligned}
U\left(\hat{a}_{i}, \mathbf{a}_{-i}\right)-U\left(a_{i}, \mathbf{a}_{-i}\right)>0 \Longrightarrow & \Phi_{g}\left(\hat{a}_{i}, \mathbf{a}_{-i}\right)-\Phi_{g}\left(a_{i}, \mathbf{a}_{-i}\right)>0, \\
& \forall a_{i}, \hat{a}_{i} \in \psi_{i} \text { and } \forall \mathbf{a}_{-i} \in \psi_{-i} .
\end{aligned}
$$

For the marginal contribution defined in Definition 5, a function $\Phi_{g}$ is a generalized ordinal potential for the game if AP $i$ obtains a better marginal contribution by performing bestresponse from an action to another one, the potential function increases with this deviation as well, i.e.,

$$
\begin{aligned}
M C_{i}^{k}-M C_{i}^{k}>0 \Longrightarrow & \Phi_{g}\left(\hat{a}_{i}, \mathbf{a}_{-i}\right)-\Phi_{g}\left(a_{i}, \mathbf{a}_{-i}\right)>0, \\
& \forall a_{i}, \hat{a}_{i} \in \psi_{i} \text { and } \forall \mathbf{a}_{-i} \in \psi_{-i},
\end{aligned}
$$

where $M C_{i}^{k}$ is the marginal contribution after performing the best response. 


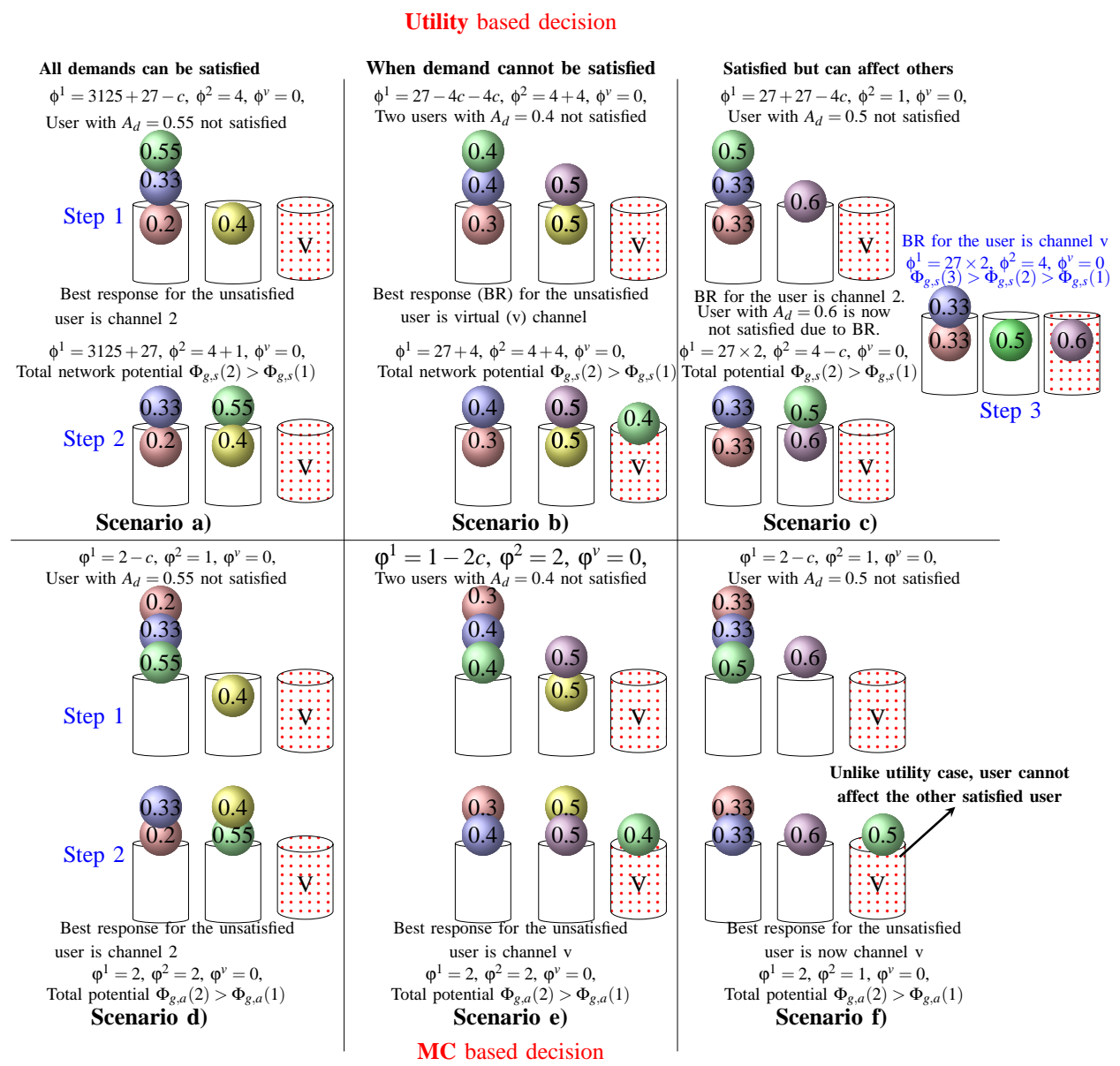

Fig. 5. Illustration of how the proposed cloud-based method enables users to converge to an NE for different scenarios under both UBR ruand marginal contributions-based decisions. Each ball represents an AP and each bin represents a channel. The number inside each ball represents an AP's airtime demand.

It was shown in [38] that every finite generalized ordinal potential game, i.e., a potential game with a finite number of players each of which has a finite set of actions, has at least one pure NE profile. In such games, an NE is a local maximum for the potential. Moreover, every finite generalized ordinal potential game has the finite best-response improvement property if the players keep performing asynchronous best response updates, i.e., the players improve their action choices by best-response one at a time, then the system will eventually reach a pure NE, as any sequence of improvement steps by players is finite, and any maximal such sequence terminates in an NE.

In the proposed UCA method, the cloud keep performing asynchronous best response updates, i.e., the cloud improves the APs' action choices one at a time, then it is easy to see that the system will eventually reach a pure NE for the generalized ordinal potential game.

Potential function under the UBR rule: Formally, the defined potential function for channel $k$ under the UBR scenario is given by:

$$
\phi^{k}(\mathbf{a})=\sum_{i \in C^{k}}\left(\left\lfloor\frac{A^{k}}{A_{d, i}}\right\rfloor\right)^{\left(\left\lfloor A^{k} / A_{d, i}\right\rfloor\right)} U\left(a_{i}, \mathbf{a}_{-i}\right),
$$

where $\mathbf{a}$ is a vector of action profiles, $C^{k}$ denotes the set of
APs which select channel $k$.

The total network potential under the UBR is given by:

$$
\Phi_{g, s}(\mathbf{a})=\sum_{k \in\{0, \mathcal{M}\}} \phi^{k}(\mathbf{a}),
$$

Potential function under the MBR rule: Formally, the defined total potential function under the MBR is given by:

$$
\Phi_{g, a}(\mathbf{a})=\sum_{k \in\{0, \mathcal{M}\}} \varphi^{k}(\mathbf{a}),
$$

where

$$
\varphi^{k}(\mathbf{a})=\sum_{i \in C^{k}} M C_{i}^{k}
$$

Next, we verify the property in Eqs. (6) and (7) for the UBR and the MBR rules, respectively. For the UBR rule, when an AP $i$ whose demand is satisfied, i.e., $U\left(a_{i}, \mathbf{a}_{-i}\right)=1$ in the current channel $k$, then it remains in the same channel since it cannot increase its utility any further. We see that in this case no change in utility corresponds to no change in total potential. When the cloud selects a new channel as a best-response for an AP $i$ which is not satisfied, then the utility of AP $i$ either increases to 1 when there is a channel available that can satisfy AP $i$ 's demand, or it increases to 0 (from $-c$ ) when there is no available channel. The best response for the no available channel case is to allocate AP $i$ to the virtual channel (null strategy). The inner sum of the 
potential function corresponding to channel $k$ (which is now unused by AP $i$ ) picks up an extra value equal to at least $\left(\left\lfloor\frac{A^{k}}{A_{d, i}}\right\rfloor\right)^{\left(\left\lfloor A^{k} / A_{d, i}\right\rfloor\right)} c$, and either increases the potential function value of the corresponding channel $k$ which is now used by AP $i$ or keeps the potential function value same, when the selected channel is the virtual channel. Thus, the left-hand side of Eq. (6) is $U\left(\hat{a}_{i}, \mathbf{a}_{-i}\right)-U\left(a_{i}, \mathbf{a}_{-i}\right)>0$, which is exactly the same as the right-hand side of Eq. (6).

Under the MBR rule, the $M C_{i}^{k}$ of AP $i$ on current channel $k$ can take one of the following values: the value of 1 , which happens when the AP $i$ has moved to $k$ which satisfies its airtime demand and also does not make any other AP unsatisfied in the same channel, value of 0 (when the AP $i$ has moved to the virtual channel), and a negative value (when the AP $i$ has moved to a channel which makes one or more other APs unsatisfied). When an AP $i$ has $M C_{i}^{k}=1$ in the current channel then it will remain in the same channel as it cannot increase its marginal contribution any further. We see that in this case no change in value corresponds to no change in total potential. When the $M C_{i}^{k}<1$ and the cloud selects a new channel as the best-response for an AP $i$, then the inner sum of the potential function corresponding to channel $k$ (which is now unused by $i$ ) strictly increases its value by at least $c$, and either increases the potential function value by one of the corresponding channel $k$ which is now used by AP $i$ or keeps the potential function value the same, when the selected channel is the virtual channel. Thus, the left-hand side of Eq. (7) is $M C_{i}^{k}-M C_{i}^{k}>0$, which is exactly the same as the righthand side of Eq. (7).

Given the generalized ordinal potential functions $\Phi g, s(\mathbf{a})$ and $\Phi g, a(\mathbf{a})$, the proof of Theorem is easy. Since a cloudbased best response increases the potential function, from finiteness, it cannot perpetually increase, and the game under the cloud-based UCA converges to an NE.

Theorem IV.2. Under the NIS scenario, when the cloud performs ordered best response updates for the APs which is the specific order of their increasing airtime demands, i.e., $A_{d, i} \geq A_{d, j} \geq, \cdots, \geq A_{d, n}$, then the proposed UCA game for both $U B R$ and MBR rules is an exact potential game. In other words, the cloud-based UCA method terminates giving an NE action profile.

Proof. A function $\Phi_{e}$ is an exact potential for the game $\mathbb{G}$, if $\forall i$

$$
\begin{aligned}
U\left(\hat{a}_{i}, \mathbf{a}_{-i}\right)-U\left(a_{i}, \mathbf{a}_{-i}\right)= & \Phi_{e}\left(\dot{a}_{i}, \mathbf{a}_{-i}\right)-\Phi_{e}\left(a_{i}, \mathbf{a}_{-i}\right), \\
& \forall a_{i}, \hat{a}_{i} \in \psi_{i} \text { and } \forall a_{-i} \in \psi_{-i} .
\end{aligned}
$$

Every finite exact potential game has the finite improvement property, if the players keep performing asynchronous better response updates, i.e., the players improve their action choices one at a time, then the system will eventually reach a pure NE. Any sequence of improvement steps by players is finite and any maximal such sequence terminates in an NE. Let a denote the action profile that is a local maximum for the potential. Then no unilateral deviation by any player can increase $\Phi_{e}(\mathbf{a})$.
Formally, the exact potential functions are given by:

$$
\Phi_{e}(\mathbf{a})=\sum_{k \in \mathcal{N}} \phi_{e}^{k}(\mathbf{a})
$$

where

$$
\phi_{e}^{k}(\mathbf{a})=\left\{\begin{array}{l}
\sum_{i \in C^{k}} U\left(a_{i}, \mathbf{a}_{-i}\right), \text { for the UBR rule, } \\
\sum_{i \in C^{k}} M C_{i}^{k}, \text { for the MBR rule. }
\end{array}\right.
$$

Next, we verify the property in Eq. (12). When the cloud performs asynchronous best response updates for APs in the order of their increasing airtime demands, $A_{d, i} \geq A_{d, j} \geq, \cdots, \geq$ $A_{d, n}$, then initially all APs are allocated to the virtual channel by the cloud. In this case, the initial potential of the system is zero. In each of the next steps where an AP's action can be updated to a new channel with the best response rules, the AP's utility/marginal contribution is increased by 1 , and we can also see that the inner sum of the potential function corresponding to channel $k$ (in which the AP is allocated due to best response) picks up an extra value equal to one. Thus, Eq. (12) is satisfied.

Given the exact potential function $\Phi_{e}(\mathbf{a})$, the proof of Theorem is easy. Since a cloud-based best response increases the potential function, from finiteness, it cannot perpetually increase, and the game under the cloud-assisted UCA converges to an NE.

One important consequence of the above two theorems is that the cloud-based method provides solution to APs which is an NE. Hence, no AP has an incentive to unilaterally deviate from the cloud-based channel selections. Under the ordered best response updates, the best response for an AP is either to be allocated to a channel which can satisfy its demand or to be allocated to the virtual channel when its demand cannot be satisfied.

\section{Convergence to an NE, Convergence time, and Information Exchange Overhead}

In the proposed algorithm, each AP $i$ and the REM repository exchange two types of low-overhead information messages: 1) static information messages, such as location information and transmission power characteristics, which are exchanged only the time when an AP is activated and registered with the REM repository; and 2) dynamic information messages, such as a change in airtime demand $A_{d, i}$ of AP $i$, change in its utility and a best response update due to change in utility. The best response updates are sent when there is a change in one or more AP's airtime channel utilization. It is easy to see that under the NIS scenario it takes $N$ best response decisions for the network to arrive at an NE. Under the RIS scenario, it takes no more than $2 N$ best response decisions for the network to arrive at an NE. The two-fold increase in the number of decisions is due to the reason that after initial random channel selections by each AP, when the cloud performs best response updates then it may happen that: 1) In the first $N$ updates, one or more APs which were unsatisfied in the initial random selection have best response updates to channels where they can be satisfied. Then some other APs which have lower airtime demands (than the best response 
updated APs) but are also unsatisfied due to initial random selection are moved to these channels and make the previously best response updated APs unsatisfied; 2) In the second $N$ updates, the unsatisfied APs are best response updated to channel where they can be satisfied or move to the virtual channel. In Fig. 5 (scenario c), we provide an illustrative example where a best response update of an AP can make other APs unsatisfied.

Remark IV.2. It is important to note that measuring convergence times of a new algorithm in real time units is nontrivial as it requires some careful attention to detail. To have some insight into the convergence performance of the proposed cloud-based UCA algorithm in real time units, we need to consider the four main operations in the proposed algorithm: 1) estimation of airtime demand for each AP. This can be achieved by using estimates of airtime consumption due to a particular application which is being used by a user device connected to an AP. Practical real time implementation complexity of such airtime estimates are shown in [39]; 2) measurement of total airtime utilization by an AP on its selected channel. In our work [33], we have demonstrated an implementation of airtime measurement module on a Xilinx FPGA. The implemented module takes only a few milliseconds to calculate airtime usage in a channel; 3) calculation of best response updates by the cloud for $N$ APs. The cloud needs to perform $N$ or no more than $2 N$ best response updates. The computation power of modern processors will take in the range of milliseconds to perform the required best response calculations; and 4) exchange of low overhead messages between APs and the cloud, such as a airtime demand/utilization, change in utility and a best response update due to change in utility. This depends on the link quality between an AP and the cloud. Typically, this can be achieved in as short time as few milliseconds.

\section{The Price of Stability and Anarchy (POS and POA)}

Up until now, we have been focussing on how APs playing together in the proposed cloud-based game arrive at an NE. However, what can be said about the quality of the NE outcome that has been reached? This is where the price of anarchy (POA) and price of stability (POS) come in. The POS and the POA with respect to NE are given, respectively, by

$$
\mathrm{POS}=\frac{\text { value of best NE }}{\text { value of optimal solution }}
$$

and

$$
\mathrm{POA}=\frac{\text { value of worst } \mathrm{NE}}{\text { value of optimal solution }} .
$$

Thus using the POS and the POA concepts, we would like to examine how good the solution represented by the best/worst $\mathrm{NE}$ of the proposed game may be, relative to the optimal solution. To do this, for the considered game, we choose to examine the optimization goal where we maximize the total utility of the system.

Observation IV.1. The considered cloud-based UCA game has $\mathrm{POS}=1$ as the optimal solution (that maximizes the total sum utility) can always be achieved in an NE point.
Observation IV.2. For the considered cloud-based game, the POA is not less than 0.5 as the optimal solution (that maximizes the total sum utility) cannot satisfy twice or more than twice as many users as compared to the solution with the worst NE. The worst NE configuration can occur when the cloud respects the initial random selection of the APs. For example, when there is considerable difference in airtime demands of the APs and the cloud respects the initial random selection of channels, then it is possible that each AP which has a low airtime utilization demand selects a separate individual channel and blocks the APs with higher demands. Let us consider the case where there are 10 available channels and 20 competing APs. Each of the 10 APs has airtime demand of $10 \%$ of a channel, and the remaining each of 10 APs demand $95 \%$ of a channel. Initially, each of the 10 APs with lower airtime demand can randomly select channels in a way that each of 10 APs selects a different channel and each gets satisfied while blocking all the other 10 APs. On the other hand, the optimal solution can allocate 10 APS with $10 \%$ airtime demand in one channel and allocate a separate channel for each of 9 APs with 95\% airtime demand. The POA in this case is 0.53 which is not less than 0.5.

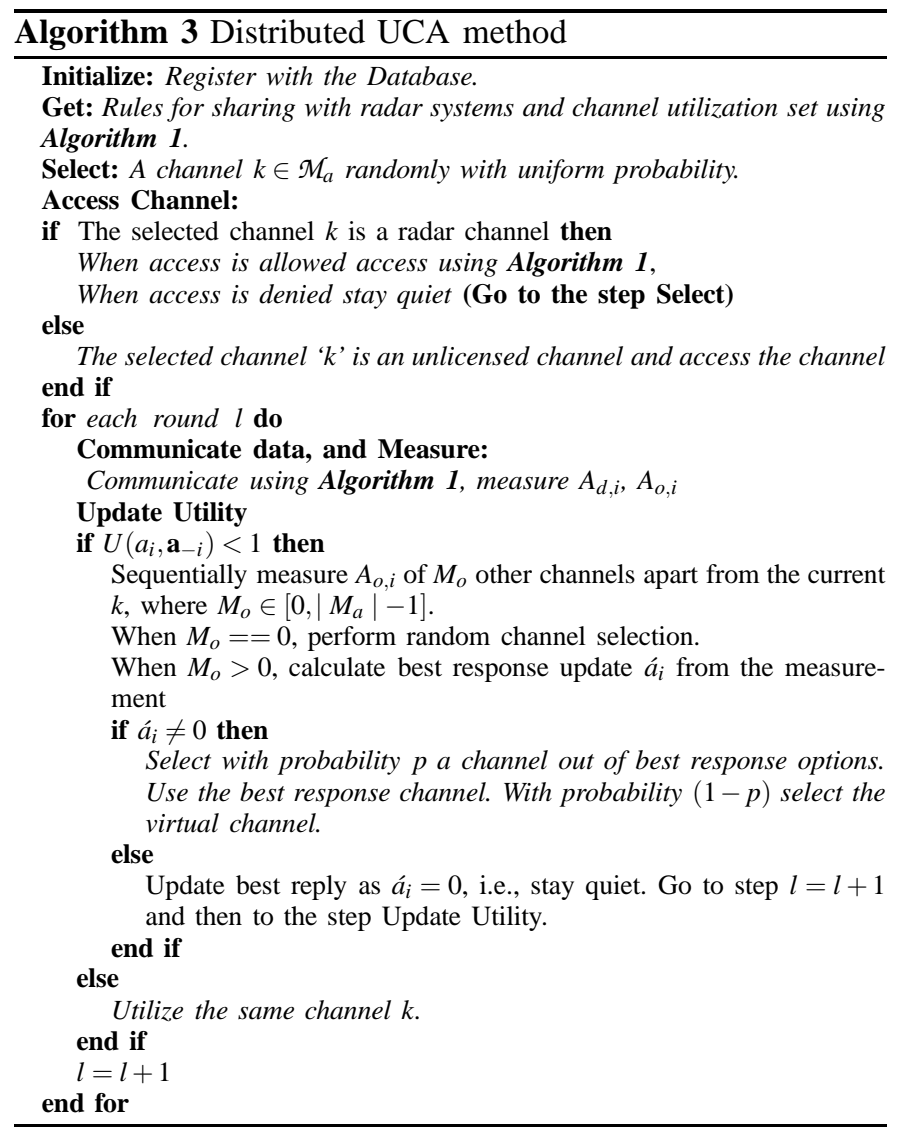

\section{Distributed UCA Method}

Different from the cloud-based method, in the distributed method each AP takes autonomous channel selection decisions, i.e., it does not take assistance from the cloud in terms of channel selections. Due to distributed channel selections, 
APs now make simultaneous channel selection decisions as compared to the cloud-based method where the cloud can perform asynchronous best response decisions on behalf of the APs.

\section{A. Algorithm}

In Algorithm 3, we present the main steps involved in our distributed UCA method in which each AP only collects information related to the radar spectrum from the database. Note that when an AP has selected an unlicensed channel it does not requires any interaction with the database. In this method, we consider two different scenarios to compute the channel selections for each AP $i$ : 1) a scenario in which an AP $i$ can only measure the airtime utilization of the channel it selects in a given round. Note that in this case, when an AP is unsatisfied in a given round, then in the next rounds, it can only perform random selection out of other channels (until it can get satisfied) as it has no airtime utilization knowledge of other channels; and 2) a scenario in which in a given round, an AP $i$ can measure the airtime utilization of the selected channel and also some other channels. In this scenario, in a given round, when an AP $i$ is not satisfied in the selected channel it measures some other channels. In the next round, it selects with probability $p \in(0,1]$ one of the channels it measured in the previous round (in which it can be satisfied), if any; otherwise, it selects the virtual channel (the null strategy).

\section{B. Condition for Convergence to an NE, POA, POS, and the Need for Regular Updates}

Remark V.1. It is easy to see that the scenario where an $A P i$ can only measure the airtime utilization of the channel it selects in a given round and performs random channel selection, the proposed distributed UCA method is not always guaranteed to converge to an NE. For example, when airtime demands of one or more APs cannot be satisfied, they can increase their utility by keep performing random channel selection/access in every round. By doing this, they can make other APs unsatisfied due to which the other APs will also perform random selection/access, as a result in some rounds, the unsatisfied APs can be satisfied.

Theorem V.1. When the APs can measure at least one other channel apart from the channel they have selected in a given round, then the proposed distributed UCA method is not guaranteed to converge to an NE for $p=1$.

Proof. Assume to the contrary that the distributed UCA method is guaranteed to converge to an NE for $p=1$. Now let us consider a 5-AP, 3-action (two real channels plus a virtual channel) distributed UCA game with $A_{d, 1}=A_{d, 2}=0.4$, $A_{d, 3}=A_{d, 4}=0.5, A_{d, 5}=0.3$, and total airtime of channels $A^{1}=A^{2}=1$ (see Fig. 6 (scenario b) for illustration). Initially, AP 1, 2, and 5 select channel 1 whereas AP 3 , and 4 select channel 2 . In channel 1 , the total airtime utilized by the three APs exceeds the total available airtime in the channel and only AP 5 can be satisfied. In channel 2, the total airtime utilized by the two APs does not exceed the total available airtime in the channel and both APs can be satisfied. In the next round, the simultaneous best response for both the unsatisfied APs is to select the virtual channel as both cannot be satisfied neither in channel 1 nor in channel 2. In round 3 of the game, both APs will find channel 1 to be their best response as both will measure the channels simultaneously and both will find channel 1 can satisfy their demand. When $p=1$, both APs will move to the channel 1 simultaneously and both will not be satisfied. The process is repeated for ever and hence an NE cannot be reached. This is contrary to the initial assumption that the distributed UCA method is guaranteed to converge to an NE for $p=1$.

When the APs can measure at least one other channel (apart from the one they have selected in a given round), then for $p<1$, the problem of entering into a cycle forever can be solved, since randomization in decisions helps multiple APs to make asynchronous channel selections. For instance, in the considered Scenario b of Fig. 6, when APs utilize $p<1$ and/or they can measure at least one channel apart from the selected channel, there is a non-zero probability that one of the two APs will first measure/select channel 1 and get satisfied, while the other AP will measure it later and will find that it cannot be satisfied, and as a result will not select the channel. In this way, the APs can arrive at an NE.

In Fig. 7a, for $N=56$ APs, we evaluate the impact of parameter $p$ on the performance of the proposed distributed UCA method in terms of average sum utility. In Fig. 7a, the APs can measure one extra channel apart from the channel they select for access. It can be seen from the figure that lowering $p$ can improve the sum utility for distributed UCA under scenarios where the total airtime utilization demands of users exceed the total airtime capacity of all the channels. However, it can be also seen from the figure that this improvement in the sum utility is only valid for some values of $p$, as for very low values of $p$, the likelihood of trying to find a new channel is significantly reduced which in turn can reduce the sum utility. The results in the figure show that for the considered scenario $p=0.5$ results in best performance in terms of average sum utility. In Fig. 7b, for $N=56$ APs, we evaluate the impact of parameter $M_{o}$, i.e., the number of measured extra channels, on the performance of the proposed distributed UCA method in terms of average sum utility. In Fig. 7b, the APs utilize $p=0.5$ for channel selection. It can be seen from the figure that when the APs can measure only the channel which they have selected to access, i.e., $M_{o}=0$, then the number of satisfied APs is around 19 APs; however, the number increases to around 33 APs for the cases where APs can measure one or two extra channels apart from the channel they access.

\section{NumericAl PERFormance ANALYsis}

\section{A. Simulation Methodology}

Through simulations, we evaluate and compare the performances of the proposed distributed and cloud-based UCA methods under the UBR and the MBR rules. We evaluate their performance in terms of average sum utility and average airtime use of satisfied APs in percentage. For the distributed UCA, we also evaluate the impact of the number of extra measured channels. Moreover, for each best response update rule, 


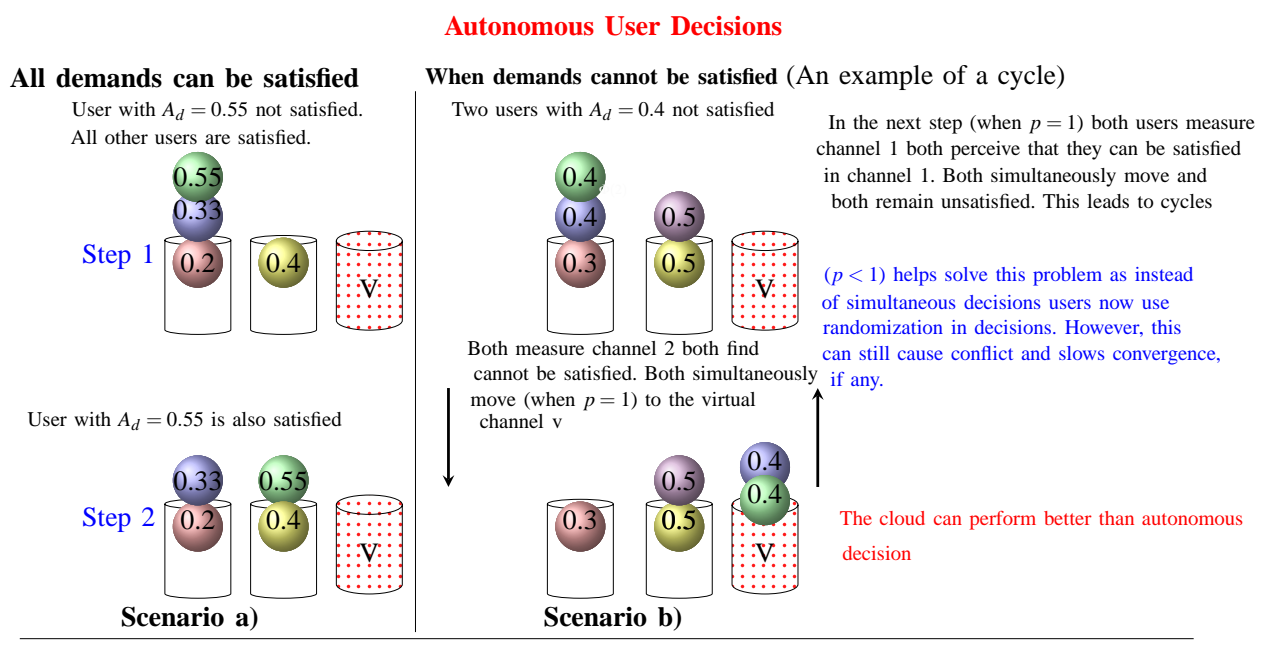

Fig. 6. Illustrations to help understanding how distributed APs when they take simultaneous decisions may not convert to an NE, and how the proposed distributed UCA method enables users to converge to an NE for different scenarios. Each ball represents an AP and each bin represents a channel. The number inside each ball represents an AP's airtime demand.

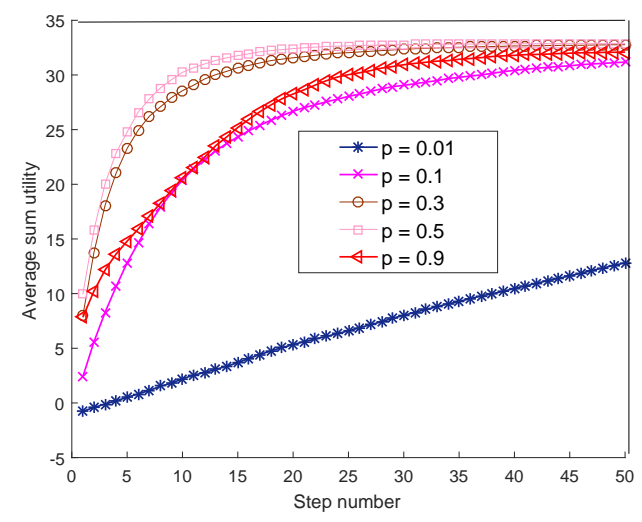

(a)

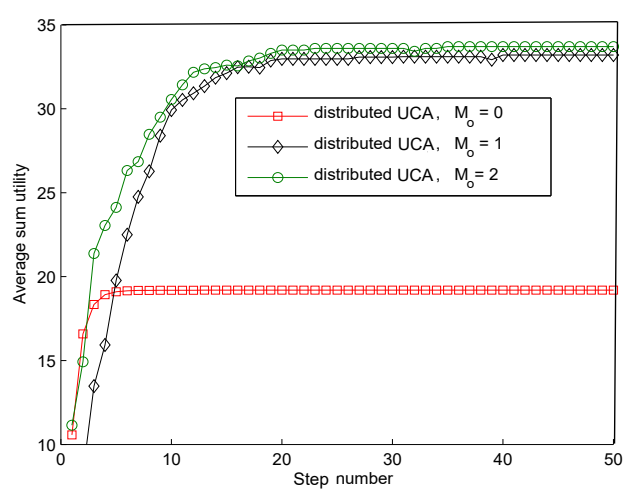

(b)

Fig. 7. a) For $N=56$ APs, we evaluate the impact of parameter $p$ on the performance of the proposed distributed UCA method in terms of average sum utility. Each AP can measure one extra channel apart from the channel they select for access. b) For $N=56$ APs, we evaluate the impact of parameter $M_{o}$, i.e., the number of measured extra channels, on the performance of the proposed distributed UCA method in terms of average sum utility. The APs utilize $p=0.5$ for channel selection. In both figures, each AP has an airtime demand $A_{d, i} \in(0,1)$. There are $M_{u}=8$ unlicensed channels, $M_{r}=4$ rotating radar channels. we also consider both RIS and NIS scenarios for performance evaluation. In all considered scenarios, each of $N$ APs has an airtime demand which takes values from $A_{d, i} \in(0,1)$. In a radar channel, a maximum of 3 APs at a given location are allowed to operate in Zone 2 of the radar system.

To evaluate how effective the proposed methods are as compared to an optimal solution, using simulations, we also compare their performance with an optimal sum utility or an optimal system welfare (based on marginal contributions) outcome in which the cloud finds the global maximum NE for the particular scenario. We will show in the results that the difference between the equilibrium outcome of the proposed methods and the global maximum is small. Note that in practice, it may not be efficient for the cloud to find the global maximum for a network of independent competing APs. This is due to the reason that unlike the proposed methods, finding the global maximum can be computationally intensive for the cloud as it requires the exploration of sheer number of possible combinations.

\section{B. Sum Utility, Airtime Utilization, and Convergence Results}

In Fig. 8a and Fig. 8b, for the considered parameters, we evaluate and compare the performance in terms of airtime utilization of the proposed cloud-based and distributed UCA method under different scenarios. We also compare their performances with an optimal solution. In Fig. 8b, Case 1 represents scenarios which take into account the impact of rejection steps, i.e., a channel selection by an AP can get rejected as its selection may have exceeded the allowed number of APs in the radar channel in a given area. Case 2 represents scenarios which ignore the impact of the rejection steps. In all the scenarios of Fig. 8a and Fig. 8b, under the distributed UCA it is considered that APs can measure one extra channel apart from the channel they select to access, and also they use $p=0.5$ for channel selection. In Fig. 8a, there are $N=26$ competing APs. It can be seen that, in terms of airtime utilization, when the cloud-based UCA utilizes MBR 


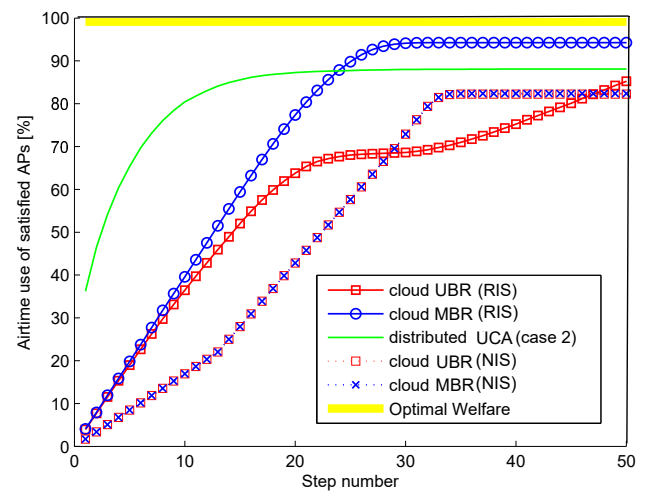

(a)

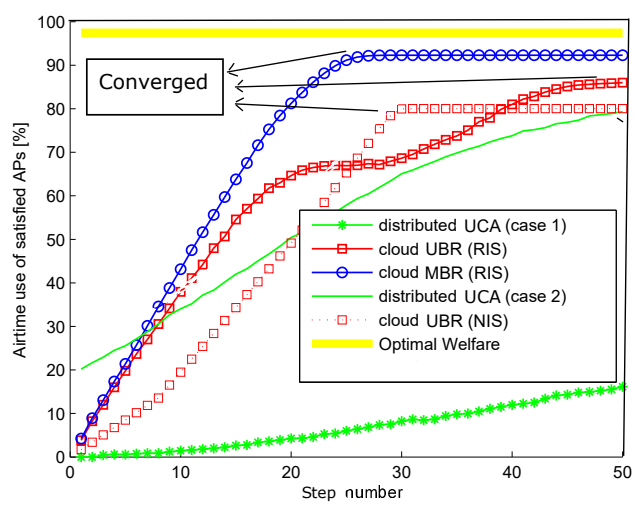

(b)

Fig. 8. Airtime utilization performance for satisfied APs under different scenarios for cloud-based and distributed UCA methods when $M_{u}=8$ unlicensed channels, $M_{r}=4$ rotating radar channels: a) When $N=26$ APs, and $M_{o}=1, p=0.5$, for distributed UCA method; b) When $N=62$ APs, and $M_{o}=1, p=0.5$, for the distributed UCA method.

rule, it outperforms utility scenarios under both cloud-based and distributed methods. Moreover, it can be also seen that the optimal solution results in $98 \%$ utilization of total available airtime of all channels, the cloud-based UCA method results in $95 \%$ utilization of total available airtime of all channels, and the distributed UCA results in $85 \%$ utilization of total available airtime of all channels.

Our results show that the RIS and the NIS scenarios can have different impact on the cloud-based channel selections. For example, it can be seen in Fig. 8a that the MBR rule under the NIS scenario gives less airtime utilization, as compared to the MBR rule under the RIS scenario. This is due to the reason that under the NIS scenario the APs with low airtime demand are allocated first, and if there are not enough available channels with sufficient airtime to satisfy all APs, the APs with high airtime demand can be blocked by the low demand APs. However, under the RIS scenario, it is possible that some high airtime demand APs are allocated first which can increase the total airtime utilization of the network. It is important to note that although this increases the total airtime utilization of the network, this can decrease the sum utility in the network as our results show in Fig. 9.

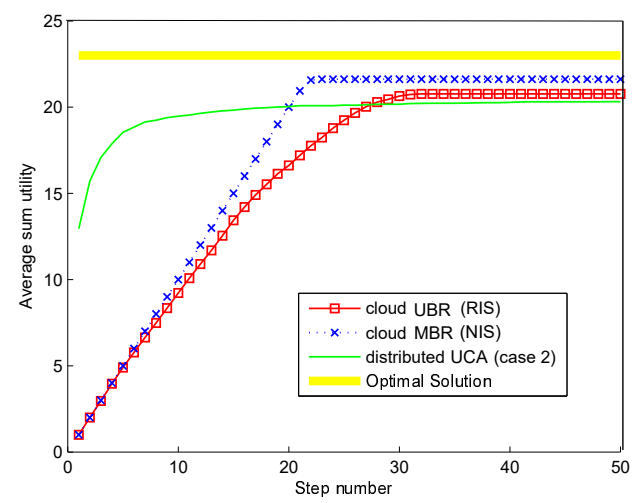

(a)

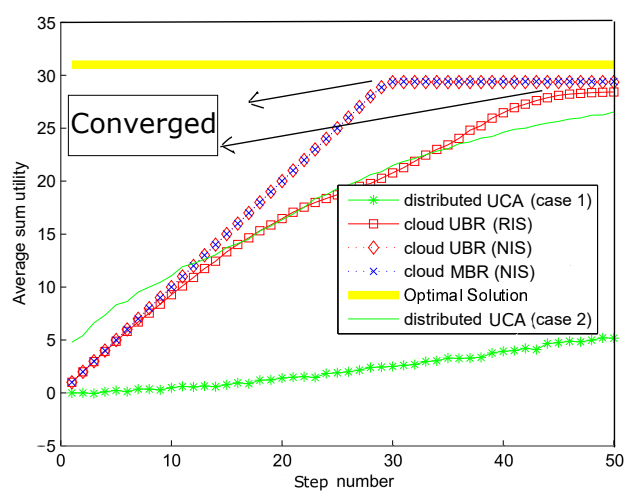

(b)

Fig. 9. Average sum utility performance under different scenarios for the cloud-based and distributed UCA methods when $M_{u}=8$ unlicensed channels, $M_{r}=4$ rotating radar channels: a) When $N=26 \mathrm{APs}$, and $M_{o}=1, p=0.5$, for distributed UCA method; b) When $N=56$ APs, and $M_{o}=1, p=0.5$, for the distributed UCA method.

It can be seen in Fig. 8 and Fig. 9 that, on one hand, in terms of airtime utilization, the method using RIS outperforms the scenarios where NIS is used. On the other hand, in terms of average sum utility, the method using NIS outperforms the scenarios where RIS is used. This is due to the reason that the NIS scenario always allows APs with less airtime utilization to be allocated first. This in turn can increase the sum utility as more more APs with low airtime utilization can be allocated, as compared to the RIS scenario where APs with high airtime utilization can also be allocated first.

In Fig. 8b, we evaluate the impact of increasing the number of APs on the airtime utilization performance. In the figure, the number of APs is increased to 62 for different scenarios. It can be seen that the increase in the number of APs has either little or no impact for the cloud-based methods whereas the distributed UCA methods' performance under case 1 is significantly reduced. This is due to the reason that while the cloud-entity has better knowledge of how many APs in a given area are allowed to operate in a rotating radar channel and how many APs have selected a channel, this information is not available to the APs using the distributed UCA. In each step, if a distributed AP selects a radar channel its selection can get rejected as its selection may have exceeded that allowed 


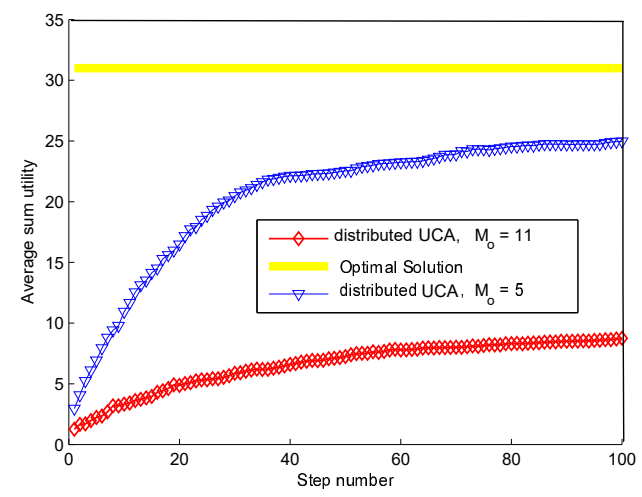

(a)

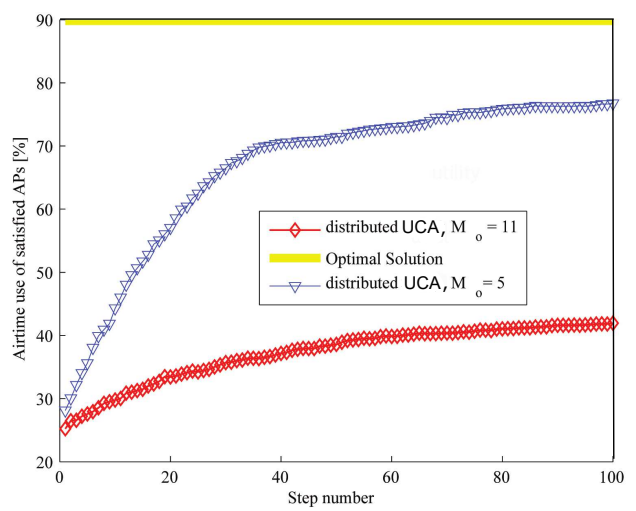

(b)

Fig. 10. Counterintuitive results for distributed UCA which show that the ability to measure many channels can decrease the average sum utility and airtime utilization, as compared to when APs can measure only few channels. Each AP can measure $M_{o}$ extra channels and each of $N=52$ APs has an airtime demand, where $A_{d, i} \in(0,1)$. There are $M_{u}=8$ unlicensed channels and $M_{r}=4$ rotating radar channels.

number of APs in the channel in a given area. In Fig. 9b, we also compare the distributed UCA's performance excluding the rejection steps (case 2 in the figure).

In Fig. 9a and Fig. 9b, we evaluate the performance of the proposed methods in terms of average sum utility. In Fig. 9a, we evaluate the performance when there are $N=26$ APs in the network, and in Fig. $9 \mathrm{~b}$ when there are $N=56$ APs. It can be seen from the figure that for $N=26$, both UBR and MBR rules for cloud-based method results in close to optimal performance. It can be also seen that for $N=56$, the performance of the distributed UCA degrades. It can also be seen in Fig. 9b and Fig. 8b that the distributed UCA method requires more steps to converge than the cloud-based method.

\section{A Counterintuitive Result}

In Fig. 10a and Fig. 10b, under the distributed UCA method, we show that when the APs can measure all the channels $\left(M_{o}=11\right.$ other channels), this reduces the average sum utility in the network and also reduces the total airtime utilization, as compared to when the APs can measure only $M_{o}=5$ other channels. This counterintuitive result stems from increased likelihood that multiple unsatisfied APs can select the same channels as their best response and is explained as follows. When there are multiple unsatisfied APs and they can measure either all or most of the available channels, then the likelihood that two or more APs select the same channel as their best response increases which in turn can make them remain unsatisfied. However, when an AP can measure only few channels, it is more likely that different APs have different best responses which in turn avoids conflict among the APs. By comparing Fig. 7b and Fig. 10a it can be seen that when the number of APs in the network is large, then for the first 50 to 100 steps, the scenario where an AP can measure only its own selected and the scenario where it can measure all the channels, both can significantly reduce the performance of the distributed UCA method. On the other hand, it can be seen that when the APs can measure few channels (even only one extra channel) the proposed distributed UCA method shows significant increase in performance.

\section{CONClusion And Future Directions}

We have designed distributed and cloud-based unified channel access (UCA) methods which enable multiple competing access points (APs) to efficiently utilize the spectrum across unlicensed and rotating radar spectrum bands. Based on spectrum usage results from our measurements campaign, we have presented a database-enabled spectrum sharing mechanism with rotating radars which is exploited by the UCA methods. We have also presented a proof-of-concept prototype implementation of database-assisted spectrum access mechanism. We have focused on a key metric of airtime utilization for the network, and have modelled the network of multiple competing APs as a UCA strategic game in which the APs attempt to maximize their utilities. We have shown that the cloud-based method takes $N$ steps to converge to an NE under certain scenarios, and takes no more than $2 N$ steps under all scenarios.

One of the extensions we envision for this work is to study the impact of scenarios where APs are allowed to use interference-aware transmit power control to negotiate power levels with their clients on a channel. Another possible extension to this work can be the study of multiuser channel access/selection in unlicensed and SA frequency bands for the scenarios where some radios are capable of full-duplex communications.

\section{REFERENCES}

[1] Qualcomm. (2013) 1000x-more-spectrum-especially-small-cells. [Online]. Available: http://www.qualcomm.com/media/documents/ 1000x-more-spectrum-especially-small-cells

[2] W. Kim, S. Ryu, J. W.-K. Hong, and Y.-J. Suh, "WLANMan: A cloudbased wireless LAN management system in ONOS controllers," in IEEE Conference on Computer Communications Workshops (INFOCOM WKSHPS), 2016, pp. 774-775.

[3] A. Patro and S. Banerjee, "Outsourcing coordination and management of home wireless access points through an open API," in IEEE Conference on Computer Communications (INFOCOM), 2015, pp. 1454-1462.

[4] CSMAC Committee, "Interference and Dynamic Spectrum Access," National Telecommunications and Information Administration (NTIA), USA, Tech. Rep., November, 2010. [Online]. Available: \{http: //www.ntia.doc.gov/legacy/advisory/spectrum/reports 
[5] M. Cotton, M. Maior, F. Sanders, E. Nelson, and D. Sicker. (March, 2012) Developing Forward Thinking Rules and Processes to Fully Exploit Spectrum Resources: An Evaluation of Radar Spectrum Use and Management. [Online]. Available: \{http://www.its.bldrdoc.gov/ publications/2669.aspx

[6] Cisco. (2014) 802.11ac: The Fifth Generation of Wi-Fi. [Online]. Available: http://www.cisco.com/c/en/us/products/collateral/ wireless/aironet-3600-series/white_paper_c11-713103.pdf

[7] G. Locke, "An Assessment of the Near-Term Viability of Accommodating Wireless Broadband Systems in the 1675-1710 $\mathrm{MHz}, 1755-1780 \mathrm{MHz}, 3500-3650 \mathrm{MHz}$, and 4200-4220 MHz, 4380$4400 \mathrm{MHz}$ Bands," National Telecommunications and Information Administration (NTIA), USA, Tech. Rep., October, 2010. [Online]. Available: $\{$ http://www.ntia.doc.gov/files/ntia/publications $\}$

[8] European Telecommunications Standards Institute, "System architecture for information exchange between different Geo-location Databases (GLDBs) enabling the operation of White Space Devices (WSDs)," Technical Specification, Tech. Rep., 2015. [Online]. Available: http://www.etsi.org/deliver

[9] Y. Khaled, H. Kokkinen, and P. Stanforth, "The State-of-the-Art in Dynamic Spectrum Access Database Technologies in 2015 and Beyond," in Dynamic Spectrum Alliance Global Summit, May 2015.

[10] Z. Khan, J. Lehtomaki, E. Hossain, S. Iellamo, R. Vuohtoniemi, and Z. Han, "IoT Connectivity in Radar Bands: A Shared Access Model Based on Spectrum Measurements," IEEE Communications Magazine, vol. 55, no. 2, pp. 88-96, February 2017.

[11] Z. Khan, J. J. Lehtomäki, R. Vuohtoniemi, E. Hossain, and L. A. DaSilva, "On opportunistic spectrum access in radar bands: Lessons learned from measurement of weather radar signals," IEEE Wireless Communications Magazine, vol. 23, no. 3, pp. 40-48, 2016.

[12] A. Hajisami, H. Viswanathan, and D. Pompili, "Cocktail Party in the Cloud: Blind Source Separation for Co-Operative Cellular Communication in Cloud RAN," in Proceedngs of IEEE 11th International Conference on Mobile Ad Hoc and Sensor Systems, Oct 2014, pp. 37-45.

[13] S. Kumar, S. Gollakota, and D. Katabi, "A cloud-assisted design for autonomous driving," in Proceedings of the First Edition of the MCC Workshop on Mobile Cloud Computing (MCC), 2012, pp. 41-46.

[14] F. Wang, J. Liu, and M. Chen, "Calms: Cloud-assisted live media streaming for globalized demands with time/region diversities," in IEEE Conference on Computer Communications (INFOCOM), 2012, pp. 199207

[15] G. Bhanage and S. Ganu, "Hybrid wireless virtualization architecture," April 2013, uS Patent App. 13/489,253. [Online]. Available: http: //www.google.com/patents/US20130100807

[16] Y. Zhang, C. Jiang, Y. Wang, J. Yuan, and J. Cao, "United Channel Assignments in Residential Environments," in IEEE Global Communications Conference (GLOBECOM), Dec 2015, pp. 1-6.

[17] C. de Souza Lima, F. Paisana, J. Ferreira de Rezende, and L. DaSilva, "A cooperative approach for dynamic spectrum access in radar bands," in International Telecommunications Symposium (ITS), August 2014, pp. $1-5$.

[18] M. Tercero, K. Sung, and J. Zander, "Temporal secondary access opportunities for WLAN in radar bands," in Proceedings of the 14th International Symposium on Wireless Personal Multimedia Communications (WPMC), 2011, pp. 1-5.

[19] D. Yuhuan and G. Veciana, "Wireless networks without edges: Dynamic radio resource clustering and user scheduling," in Proceedings of IEEE Conference on Computer Communications (INFOCOM), April 2014, pp. 1321-1329.

[20] S. Andreev, M. Gerasimenko, O. Galinina, Y. Koucheryavy, N. Himayat, S. Yeh, and S. Talwar, "Intelligent access network selection in converged multi-radio heterogeneous networks," IEEE Wireless Communications, vol. 21, no. 6, pp. 86-96, 2014.

[21] J. Singh, "Resource allocation in coordinated and un-coordinated wireless systems with greedy or non-greedy users," Ph.D. dissertation, Rutgers, The State University of New Jersey, USA, 2007.

[22] S. Deb, P. Gupta, K. Nagaraj, and V. Srinivasan, "An Agile and Efficient MAC for Wireless Access over TV Whitespaces," IEEE Transactions on Mobile Computing, vol. 14, no. 1, pp. 42-57, Jan 2015.

[23] Y. Xu, Y. Xu, and A. Anpalagan, "Database-assisted spectrum access in dynamic networks: A distributed learning solution," IEEE Access, vol. 3, pp. 1071-1078, 2015.

[24] X. Chen, X. Gong, L. Yang, and J. Zhang, "A social group utility maximization framework with applications in database assisted spectrum access," in IEEE Conference on Computer Communications (INFOCOM), 2014, pp. 1959-1967.
[25] J. Herzen, R. Merz, and P. Thiran, "Distributed spectrum assignment for home WLANs," in Proceeedings of IEEE Conference on Computer Communications (INFOCOM), 2013, pp. 1573-1581.

[26] Y. Xu, J. Wang, Y. Xu, L. Shen, Q. Wu, and A. Anpalagan, "Centralizeddistributed spectrum access for small cell networks: A cloud-based game solution," arXiv preprint arXiv:1502.06670, 2015.

[27] A. Vonnagy. (August, 2015) Understanding WiFi's Breaking Point to Design Better WLANs. [Online]. Available: \{http://community.arubanetworks.com/t5/Technology-Blog/ Push-It-To-The-Limit-Understand-Wi-Fi-s-Breaking-Point-to-Design/ ba-p/241378\}

[28] Aerohive. (2015) Optimizing Network and Client Performance Through Dynamic Airtime Scheduling. [Online]. Available: \{http://www.aerohive.com/pdfs/Aerohive-Whitepaper-Dynamic_ Airtime_Scheduling.pdf $\}$

[29] G. Zhou, "System and method for wireless local area network airtime fairness," Patent US8 818 293, September, 2013.

[30] Cisco. (2015) Cisco Air Time Fairness. [Online]. Available: http://www.cisco.com/c/en/us/td/docs/wireless/controller/8-1/ configuration-guide/b_cg81/b_cg81_chapter_010000111.pdf

[31] F. H. Sanders, E. F. Drocella, and R. L. Sole, "Using On-Shore Detected Radar Signal Power for Interference Protection of OffShore Radar Receivers," National Telecommunications and Information Administration (NTIA), USA, Tech. Rep., March, 2016. [Online]. Available: \{https://www.its.bldrdoc.gov/publications/2828.aspx

[32] E. Drocella, J. Richards, R. Sole, F. Najmy, A. Lundy, and P. McKenna, "3.5 GHz Exclusion Zone Analyses and Methodology," National Telecommunications and Information Administration (NTIA), USA, Tech. Rep., June, 2015. [Online]. Available: $\{$ https://www.ntia.doc.gov/ report/2015/35-ghz-exclusion-zone-analyses-and-methodology

[33] Z. Khan, J. Lehtomaki, E. Hossain, M. Latva-aho, and A. Marshall, "Design and prototype of a multiobjective environment sensing capability for shared access with rotating radars," IEEE Wireless Communications Magazine, under review, pp. 1-14, 2017.

[34] Rice University WARP project. [Online]. Available: \{http://warp.rice. $\mathrm{edu} /\}$

[35] A. Argento, M. Cesana, N. Gatti, and I. Malanchini, "A game theoretical study of access point association in wireless mesh networks," Computer Communications, vol. 35, no. 5, pp. 541 - 553, 2012.

[36] P. Bahl, R. Chandra, T. Moscibroda, R. Murty, and M. Welsh, "White Space Networking with WiFi Like Connectivity," in Proceedings of the ACM Conference on Data Communication (SIGCOMM), 2009, pp. 2738.

[37] Y. Zhao, "Enabling cognitive radios through radio environment maps," Ph.D. dissertation, Virginia Tech, USA, 2007.

[38] D. Monderer and L. S. Shapley, "Potential games," Games and Economic Behavior, vol. 14, no. 1, pp. 124 - 143, 1996. [Online]. Available: http://www.sciencedirect.com/science/article/pii/S0899825696900445

[39] Aerohive Networks, Inc., "High-DEnsity WiFi Design Principles," Aerohive Networks, Tech. Rep., 2012. [Online]. Available: \{http://media.aerohive.com/documents/2034844328_ Aerohive-Whitepaper-Hi-Density_Principles.pdf 